Elect. Comm. in Probab. 16 (2011), 213-225

\title{
ASYMPTOTIC FIRST EXIT TIMES OF THE CHAFEE-INFANTE EQUA- TION WITH SMALL HEAVY-TAILED LÉVY NOISE
}

\author{
ARNAUD DEBUSSCHE \\ ENS Cachan, Antenne de Bretagne, Avenue Robert Schumann, F 35170 Bruz \\ email: arnaud.debussche@bretagne.ens-cachan.fr \\ MICHAEL HÖGELE ${ }^{1}$ \\ Universität Potsdam, Institut für Mathematik, Am Neuen Palais 10, D 14465 Potsdam \\ email: hoegele@math . uni-potsdam.de \\ PETER IMKELLER \\ Humboldt-Universität zu Berlin, Institut für Mathematik, Rudower Chaussee 25, D 12489 Berlin \\ email: imkeller@math.hu-berlin.de
}

Submitted 11/12/2010, accepted in final form 12/03/2011

AMS 2000 Subject classification: 60H15; 60G51; 35R69; 60J75; 92F99

Keywords: stochastic reaction diffusion equation with heavy-tailed Lévy noise; first exit times; regularly varying Lévy process; small noise asymptotics

\section{Abstract}

This article studies the behavior of stochastic reaction-diffusion equations driven by additive regularly varying pure jump Lévy noise in the limit of small noise intensity. It is shown that the law of the suitably renormalized first exit times from the domain of attraction of a stable state converges to an exponential law of parameter 1 in a strong sense of Laplace transforms, including exponential moments. As a consequence, the expected exit times increase polynomially in the inverse intensity, in contrast to Gaussian perturbations, where this growth is known to be of exponential rate.

\section{Introduction}

Energy balance models with random perturbations may provide crucial probabilistic insight into paleoclimatological phenomena on a conceptual level (see [1], [13]). Following the suggestion by [5] and [6], in [14] the authors determine asymptotic first exit times for one-dimensional heavy-tailed Lévy diffusions from reduced domains of attraction in the limit of small intensity. Exponential moments not being available, the arguments leading to these results do not employ large deviations methods, as opposed to [9]. [14] shows that in contrast to the case of Gaussian

\footnotetext{
${ }^{1}$ RESEARCH SUPPORTED BY THE IRTG SMCP BERLIN-ZURICH AND THE BERLIN MATHEMATICAL SCHOOL
} 
diffusions the expected first exit times are polynomial in terms of the inverse intensity. In this article these finite dimensional results are generalized to a class of reaction-diffusion equations, the prototype of which is the Chafee-Infante equation.

Let $X^{\varepsilon}$ be the solution process of the stochastic Chafee-Infante equation driven by $\varepsilon L$, an additive regularly varying Lévy noise of index $\alpha \in(0,2)$ at intensity $\varepsilon>0$. In this work we study the laws of the asymptotic first exit times $\tau^{ \pm}(\varepsilon)$ of $X^{\varepsilon}$ from a (slightly reduced) domain of attraction of the deterministic Chafee-Infante equation $u=X^{0}$ in the small noise limit $\varepsilon \rightarrow 0$. We show that there exists a polynomial scale $\lambda^{ \pm}(\varepsilon) \approx \varepsilon^{\alpha}$ linking the Lévy measure of $L$ and the domain of attraction

of $u$, such that $\lambda^{ \pm}(\varepsilon) \tau^{ \pm}(\varepsilon) \stackrel{d}{\rightarrow} \bar{\tau}$, where $\bar{\tau} \sim \operatorname{EXP}(1)$. In particular $\mathbb{E}\left[\tau^{ \pm}(\varepsilon)\right] \approx \frac{1}{\varepsilon^{\alpha}}$ in the limit of small $\varepsilon$.

This contrasts sharply with corresponding results in the case of Gaussian perturbation [8], where large deviations estimates in the spirit of Freidlin and Ventsell are used to show exponential growth of first exit times in the limit of small $\varepsilon$. Applied in a climatological context, the Chafee-Infante equation is able to describe energy-balance based reaction-diffusion equations, in which latitudinal heat transport is possible, and states of the system can be seen as temperature distributions on the interval between south and north pole. In this setting, our result suggests a probabilistic interpretation of fast transitions between different climate states corresponding to the stable equilibria observed in ice core time series of temperature proxies of [3].

In the following sections we outline the partially tedious and complex arguments needed to describe the asymptotic properties of the exit times. Detailed proofs in particular of the more technical parts are given in the forthcoming [4].

\section{Preliminaries and the main result}

Let $H=H_{0}^{1}(0,1)$ be normed by $\|u\|:=|\nabla u|$ for $u \in H$, where $|\cdot|$ is the norm in $L^{2}(0,1)$ and $\mathscr{C}_{0}([0,1])$ the space of continuous functions $u:[0,1] \rightarrow \mathbb{R}$ with $u(0)=u(1)=0$ and the supremum norm $|\cdot|_{\infty}$. Since $|u| \leqslant|u|_{\infty} \leqslant\|u\|$ for $u \in H$ we obtain the continuous injections $L^{2}(0,1) \hookrightarrow \mathscr{C}_{0}(0,1) \hookrightarrow H$. Denote by $M_{0}(H)$ the class of all Radon measures $v: \mathscr{B}(H) \rightarrow[0, \infty]$ satisfying

$$
v(A)<\infty \quad \Leftrightarrow \quad A \in \mathscr{B}(H), 0 \notin \bar{A} .
$$

Let $(L(t))_{t \geqslant 0}$ be a càdlàg version of a pure jump Lévy process in $H$ with a symmetric Lévy measure $v \in M_{0}(H)$ on its Borel $\sigma$-algebra $\mathscr{B}(H)$ satisfying

$$
\int_{H} \min \left\{1,\|y\|^{2}\right\} v(\mathrm{~d} y)<\infty \quad \text { and } \quad v(A)=v(-A), \quad A \in \mathscr{B}(H), 0 \notin \bar{A},
$$

and which is regularly varying with index $\alpha=-\beta \in(0,2)$ and limiting measure $\mu \in M_{0}(H)$. For a more comprehensive account we refer to [2] and [12].

Fix $\pi^{2}<\lambda \neq(\pi n)^{2}$ and $f(z)=-\lambda\left(z^{3}-z\right)$ for $z \in \mathbb{R}$. The object of study of this article is the behaviour of the solution process $X^{\varepsilon}$ in $H$ of the following system for small $\varepsilon>0$. For $x \in H$ consider

$$
\begin{aligned}
\frac{\partial}{\partial t} X^{\varepsilon}(t, \zeta) & =\frac{\partial^{2}}{\partial \zeta^{2}} X^{\varepsilon}(t, \zeta)+f\left(X^{\varepsilon}(t, \zeta)\right)+\varepsilon \dot{L}(t, \zeta), & & t>0, \zeta \in[0,1], \\
X^{\varepsilon}(t, 0) & =X^{\varepsilon}(t, 1)=0, & & t>0, \\
X^{\varepsilon}(0, \zeta) & =x(\zeta), & & \zeta \in[0,1] .
\end{aligned}
$$


We summarize some results for the solution $u(t ; x)=X^{0}(t ; x)$ of the deterministic Chafee-Infante equation (ChI). It is well-known that the solution flow $(t, x) \mapsto u(t ; x)$ is continuous in $t$ and $x$ and defines a dynamical system in $H$. Furthermore the solutions are extremely regular for any positive time, i.e. $u(t) \in \mathscr{C}^{\infty}(0,1)$ for $t>0$. The attractor of (ChI) is explicitly known to be contained in the unit ball with respect to the norm $|\cdot|_{\infty}$ (see for instance [7], Chapter 5.6).

Proposition 2.1. For $\lambda>0$ denote by $\mathscr{E}^{\lambda}$ the set of fixed points of (ChI). Then for any $\lambda>0$ and initial value $x \in H$ there exists a stationary state $\psi \in \mathscr{E}^{\lambda}$ of the system (ChI) such that

$$
\lim _{t \rightarrow \infty} u(t ; x)=\psi
$$

Furthermore if $\pi^{2}<\lambda \neq(k \pi)^{2}, k \in \mathbb{N}$, there are two stable fixed points and all elements of $\mathscr{E}^{\lambda}$ are hyperbolic. In addition, the stable and the unstable manifolds of any unstable fixed point of $\mathscr{E}^{\lambda}$ intersect transversally.

This relies on the fact that there is an energy functional, which may serve as a Lyapunov function for the system. A proof of the first part can be found in [8], [11], and of the second part in [10].

Definition 2.2. For $\lambda>\pi^{2}$ the solution of system (ChI) has two stable stationary states denoted by $\phi^{+}$and $\phi^{-}$. The full domains of attraction are given by

$$
D^{ \pm}:=\left\{x \in H \mid \lim _{t \rightarrow \infty} u(t ; x)=\phi^{ \pm}\right\}, \quad \text { and } \quad D_{0}^{ \pm}:=D^{ \pm}-\phi^{ \pm},
$$

and the separatrix by

$$
\mathscr{S}:=H \backslash\left(D^{+} \cup D^{-}\right)
$$

Due to the Morse-Smale property the separatrix is a closed $\mathscr{C}^{1}$-manifold without boundary in $H$ of codimension 1 separating $D^{+}$from $D^{-}$, and containing all unstable fixed points. For more refined results we refer to [17] and references therein.

Definition 2.3. Writing $B_{\delta}(x)$ for the ball of radius $\delta>0$ in $H$ with respect to the $|\cdot|_{\infty}-$ norm centered at $x$, denote for $\delta_{1}, \delta_{2}, \delta_{3} \in(0,1)$

$$
\begin{aligned}
D^{ \pm}\left(\delta_{1}\right) & :=\left\{x \in D^{ \pm} \mid \cup_{t \geqslant 0} B_{\delta_{1}}(u(t ; x)) \subset D^{ \pm}\right\}, \\
D^{ \pm}\left(\delta_{1}, \delta_{2}\right) & :=\left\{x \in D^{ \pm} \mid \cup_{t \geqslant 0} B_{\delta_{2}}(u(t ; x)) \subset D^{ \pm}\left(\delta_{1}\right)\right\}, \\
D^{ \pm}\left(\delta_{1}, \delta_{2}, \delta_{3}\right) & :=\left\{x \in D^{ \pm} \mid \cup_{t \geqslant 0} B_{\delta_{3}}(u(t ; x)) \subset D^{ \pm}\left(\delta_{1}, \delta_{2}\right)\right\} .
\end{aligned}
$$

For $\gamma \in(0,1)$ the sets $\tilde{D}^{ \pm}\left(\varepsilon^{\gamma}\right):=D^{ \pm}\left(\varepsilon^{\gamma}, \varepsilon^{2 \gamma}\right)$ and $D^{ \pm}\left(\varepsilon^{\gamma}, \varepsilon^{2 \gamma}, \varepsilon^{2 \gamma}\right)$ will be of particular importance. We define the reshifted domains of attraction by

$$
\begin{aligned}
D_{0}^{ \pm}\left(\delta_{1}\right) & :=D^{ \pm}\left(\delta_{1}\right)-\phi^{ \pm}, \\
D_{0}^{ \pm}\left(\delta_{1}, \delta_{2}\right) & :=D^{ \pm}\left(\delta_{1}, \delta_{2}\right)-\phi^{ \pm}, \\
D_{0}^{ \pm}\left(\delta_{1}, \delta_{2}, \delta_{3}\right) & :=D^{ \pm}\left(\delta_{1}, \delta_{2}, \delta_{3}\right)-\phi^{ \pm},
\end{aligned}
$$

and the following neighborhoods of the separatrix $\mathscr{S}$

$$
\begin{aligned}
& \tilde{D}^{0}\left(\varepsilon^{\gamma}\right):=H \backslash\left(\tilde{D}^{+}\left(\varepsilon^{\gamma}\right) \cup \tilde{D}^{-}\left(\varepsilon^{\gamma}\right)\right), \\
& D_{0}^{*}\left(\varepsilon^{\gamma}\right):=\left(D_{0}^{ \pm}(\varepsilon) \backslash D_{0}\left(\varepsilon^{\gamma}, \varepsilon^{2 \gamma}\right)\right)+B_{\varepsilon^{2 \gamma}}(0) .
\end{aligned}
$$


In [4] it is shown that the union over all $\varepsilon>0$ for each of the sets $D^{ \pm}\left(\varepsilon^{\gamma}\right), \tilde{D}^{ \pm}\left(\varepsilon^{\gamma}\right)$ and $D^{ \pm}\left(\varepsilon^{\gamma}, \varepsilon^{2 \gamma}, \varepsilon^{2 \gamma}\right)$ exhausts $D^{ \pm}$. Furthermore $D^{ \pm}\left(\varepsilon^{\gamma}\right)$ and $\tilde{D}^{ \pm}\left(\varepsilon^{\gamma}\right)$ are positively invariant under the deterministic solution flow, and $\tilde{D}^{ \pm}\left(\varepsilon^{\gamma}\right)+B_{\varepsilon^{2 \gamma}}(0) \subset D^{ \pm}\left(\varepsilon^{\gamma}\right)$ and $D^{ \pm}\left(\varepsilon^{\gamma}, \varepsilon^{2 \gamma}, \varepsilon^{2 \gamma}\right)+B_{\varepsilon^{2 \gamma}}(0) \subset \tilde{D}^{ \pm}\left(\varepsilon^{\gamma}\right)$.

Proposition 2.4. Given the Chafee-Infante parameter $\pi^{2}<\lambda \neq(k \pi)^{2}$ for all $k \in \mathbb{N}$ there exist a finite time $T_{\text {rec }}=T_{\text {rec }}(\lambda)>0$ and a constant $\kappa=\kappa(\lambda)>0$, which satisfy the following. For each $\gamma>0$ there is $\varepsilon_{0}=\varepsilon_{0}(\gamma)>0$, such that for all $0<\varepsilon \leqslant \varepsilon_{0}, T_{\text {rec }}+\kappa \gamma|\ln \varepsilon| \leqslant t$ and $x \in D^{ \pm}\left(\varepsilon^{\gamma}\right)$

$$
\left|u(t ; x)-\phi^{ \pm}\right|_{\infty} \leqslant(1 / 2) \varepsilon^{2 \gamma} .
$$

This results relies on the hyperbolicity of the fixed points and the fine dynamics of the deterministic solution flow. In [4] it is proved in the stronger Hilbert space topology of $H$. The preceding theorem follows then as a corollary.

We denote the jump increment of $L$ at time $t \geqslant 0$ by $\Delta_{t} L:=L(t)-L(t-)$, and decompose the process $L$ for $\rho \in(0,1)$ and $\varepsilon>0$ in the following way. We call $\eta^{\varepsilon}$ the "large jump" compound Poisson process with intensity $\beta_{\varepsilon}:=v\left(\varepsilon^{-\rho} B_{1}^{c}(0)\right)$ and jump probability measure $v\left(\cdot \cap \varepsilon^{-\rho} B_{1}^{c}(0)\right) / \beta_{\varepsilon}$, and the complementary "small jump" process $\xi^{\varepsilon}:=L-\eta^{\varepsilon}$. The process $\xi^{\varepsilon}$ is a mean zero martingale in $H$ thanks to the symmetry of $v$ with finite exponential moments. We define the jump times of $\eta^{\varepsilon}$ as

$$
T_{0}:=0, \quad T_{k}:=\inf \left\{t>T_{k-1} \mid\left\|\Delta_{t} L\right\|>\varepsilon^{-\rho}\right\}, \quad k \geqslant 1,
$$

and the times between successive large jumps of $\eta_{t}^{\varepsilon}$ recursively as $t_{0}=0$ and $t_{k}:=T_{k}-T_{k-1}$, for $k \geqslant 1$. Their laws $\mathscr{L}\left(t_{k}\right)$ are exponential $\operatorname{EXP}\left(\beta_{\varepsilon}\right)$. We shall denote the $k$-th large jump by $W_{0}=0$ and $W_{k}=\Delta_{T_{k}} L$ for $k \geqslant 1$.

Proposition 2.5. For any mean zero $L^{2}(\mathbb{P} ; H)$-martingale $\xi=(\xi(t))_{t \geqslant 0}, T>0$, and initial value $x \in H$ equation (2.1) driven by $\varepsilon \xi$ instead of $\varepsilon L$ has a unique càdlàg mild solution $\left(Y^{\varepsilon}(t ; x)\right)_{t \in[0, T]}$. The solution process $Y^{\varepsilon}$ induces a homogeneous Markov family satisfying the Feller property.

A proof can be found in [16], Chapter 10. By localization this notion of solution is extended to the heavy-tailed process $L$. In [4] this will be carried out in detail.

Corollary 2.6. For $x \in H$ equation (2.1) has a càdlàg mild solution $\left(X^{\varepsilon}(t ; x)\right)_{t \geqslant 0}$, which satisfies the strong Markov property.

Definition 2.7. For $\gamma \in(0,1), \varepsilon>0$, and the càdlàg mild solution $X^{\varepsilon}(\cdot ; x)$ of (2.1) with initial position $x \in \tilde{D}^{ \pm}\left(\varepsilon^{\gamma}\right)$ we define the first exit time from the reduced domain of attraction

$$
\tau_{x}^{ \pm}(\varepsilon):=\inf \left\{t>0 \mid X^{\varepsilon}(t ; x) \notin D^{ \pm}\left(\varepsilon^{\gamma}\right)\right\} .
$$

We now introduce the following two hypotheses, which will be required in our main theorem. They are natural conditions on the regularly varying Lévy measure $v$ with respect to the underlying deterministic dynamics in terms of its limit measure $\mu$. See [12] for the relationship between $v$ and $\mu$, and (2.9) below for the particular scaling function $\frac{1}{\epsilon}$ needed here.

(H.1) Non-trivial transitions: $\mu\left(\left(D_{0}^{ \pm}\right)^{c}\right)>0$.

(H.2) Non-degenerate limiting measure: For $\alpha \in(0,2)$ and $\Gamma>0$ according to Proposition 3.4 let

$$
0<\Theta<\frac{2-\alpha}{2 \alpha}, \rho \in\left(\frac{1}{2}, \frac{2-\alpha}{2-(1-\Theta) \alpha}\right), 0<\gamma<\frac{(2-\alpha)(1-\rho)-\Theta \alpha \rho}{2(\Gamma+2)} .
$$

For $k= \pm$ and $\eta>0$ there is $\varepsilon_{0}>0$ such that for all $0<\varepsilon \leqslant \varepsilon_{0}$

$$
\mu\left(H \backslash\left(\left(D^{+}\left(\varepsilon^{\gamma}, \varepsilon^{2 \gamma}, \varepsilon^{2 \gamma}\right) \cup D^{-}\left(\varepsilon^{\gamma}, \varepsilon^{2 \gamma}, \varepsilon^{2 \gamma}\right)\right)+B_{\varepsilon^{2 \gamma}}(0)\right)-\phi^{k}\right)<\eta .
$$


While (H.1) ensures that there actually are transitions also by "large" jumps with positive probability, (H.2) implies that the slow deterministic dynamics close to the separatrix does not distort the generic exit scenario of $X^{\varepsilon}$. For comparable finite dimensional situations with absolutely continuous Lévy measure $v \ll d x$ these hypotheses are always satisfied.

For $\varepsilon>0$ we define the characteristic rate of the system (2.1) by

$$
\lambda^{ \pm}(\varepsilon):=v\left(\frac{1}{\varepsilon}\left(D_{0}^{ \pm}\right)^{c}\right) .
$$

According to [2] and [12] for $v$ chosen above there is a slowly varying function $\ell_{v}=\ell:(0, \infty) \rightarrow$ $(0, \infty)$ such that for all $\varepsilon>0$

$$
\lambda^{ \pm}(\varepsilon)=\varepsilon^{\alpha} \ell\left(\frac{1}{\varepsilon}\right) \mu\left(\left(D_{0}^{ \pm}\right)^{c}\right), \quad \text { and } \quad \beta_{\varepsilon}=\varepsilon^{\alpha \rho} \ell\left(\frac{1}{\varepsilon^{\rho}}\right) \mu\left(B_{1}^{c}(0)\right) .
$$

We may now state the main theorem.

Theorem 2.8. Given the Chafee-Infante parameter $\pi^{2}<\lambda \neq(k \pi)^{2}$ for all $k \in \mathbb{N}$, we suppose that Hypotheses (H.1) and (H.2) are satisfied. Then for any $\theta>-1$

$$
\lim _{\varepsilon \rightarrow 0+} \mathbb{E}\left[\sup _{x \in \tilde{D}^{ \pm}\left(\varepsilon^{\gamma}\right)} \exp \left(-\theta \lambda^{ \pm}(\varepsilon) \tau_{x}^{ \pm}(\varepsilon)\right)\right]=\frac{1}{1+\theta}
$$

The supremum in the formula can be replaced by the infimum.

The theorem states that in the limit of small $\varepsilon$, suitably renormalized exit times from reduced domains of attraction have unit exponential laws.

\section{The Small Deviation of the Small Noise Solution}

This section is devoted to a small deviations' estimate. It quantifies the fact, that in the time interval between two adjacent large jumps the solution of the Chafee-Infante equation perturbed by only the small noise component deviates from the solution of the deterministic equation by only a small $\varepsilon$-dependent quantity, with probability converging to 1 in the small noise limit $\varepsilon \rightarrow 0$. Define the stochastic convolution $\xi^{*}$ with respect to the small jump part $\xi^{\varepsilon}$ by $\xi^{*}(t)=\int_{0}^{t} S(t-$ $s) \mathrm{d} \xi^{\varepsilon}(s)$ for $t \geqslant 0$ (see [16]). In order to control the deviation for $Y^{\varepsilon}-u$ for small $\varepsilon>0$, we decompose $Y^{\varepsilon}=u+\varepsilon \xi^{*}+R^{\varepsilon}$. By standard methods we obtain in [4] the following lemmas.

Lemma 3.1. For $\rho \in(0,1), \gamma>0, p>0$ and $0<\Theta<1$ there are constants $C>0$ and $\varepsilon_{0}>0$ such that for $0<\varepsilon \leqslant \varepsilon_{0}$ and $T \geqslant 0$

$$
\mathbb{P}\left(\sup _{t \in[0, T]}\left\|\varepsilon \xi_{t}^{*}\right\| \geqslant \varepsilon^{p}\right) \leqslant C T \varepsilon^{2-2 p-(2-(1-\Theta) \alpha) \rho} .
$$

Define for $T>0, \Gamma>0$ and $\gamma>0$ the small convolution event

$$
\mathscr{E}_{T}\left(\varepsilon^{(\Gamma+2) \gamma}\right):=\left\{\sup _{r \in[0, T]}\left\|\varepsilon \xi^{*}(r)\right\|<\varepsilon^{(\Gamma+2) \gamma}\right\} \quad \varepsilon>0 .
$$

By perturbation arguments, the stability of $\phi^{ \pm}$, Proposition 2.4 and Lemma 3.1 we may estimate the remainder term $R^{\varepsilon}$ for small $\varepsilon$. 
Lemma 3.2. There is a constant $\Gamma>0$ such that for $\rho \in(1 / 2,1), \gamma>0$, there exists $\varepsilon_{0}>0$ such that for $0<\varepsilon \leqslant \varepsilon_{0}, T>0, x \in D^{ \pm}\left(\varepsilon^{\gamma}\right)$ on the event $\mathscr{E}_{T}\left(\varepsilon^{(\Gamma+2) \gamma}\right)$ we have the estimate

$$
\sup _{t \in[0, T]}\left|R^{\varepsilon}(t ; x)\right|_{\infty} \leqslant \frac{1}{4} \varepsilon^{2 \gamma} .
$$

We next combine Proposition 2.4, Lemma 3.1 and Lemma 3.2, to obtain the following proposition on small deviations on deterministic time intervals.

Proposition 3.3. There is a constant $\Gamma>0$ such that for $0<\alpha<2$ given the conditions

$$
0<\Theta<\frac{2-\alpha}{\alpha}, \quad \rho \in\left(1 / 2, \frac{2-\alpha}{2-(1-\Theta) \alpha}\right), \quad 0<\gamma<\frac{(2-\alpha)(1-\rho)-\Theta \alpha \rho}{2(\Gamma+2)},
$$

there exist $\varepsilon_{0}>0$ and $C>0$ such that for any $T>0,0<\varepsilon \leqslant \varepsilon_{0}$ and $x \in D^{ \pm}\left(\varepsilon^{\gamma}\right)$

$$
\mathbb{P}\left(\sup _{s \in[0, T]}\left|Y^{\varepsilon}(s ; x)-u(s ; x)\right|_{\infty} \geqslant(1 / 2) \varepsilon^{2 \gamma}\right) \leqslant C T \varepsilon^{2-2(\Gamma+2) \gamma-(2-(1-\Theta) \alpha) \rho} .
$$

This can be generalized to the first jump time $T_{1}$ replacing $T$.

Proposition 3.4. There is a constant $\Gamma>0$ such that for $0<\alpha<2$ given the conditions

$$
0<\Theta<\frac{2-\alpha}{\alpha}, \quad \rho \in\left(1 / 2, \frac{2-\alpha}{2-(1-\Theta) \alpha}\right), \quad 0<\gamma<\frac{(2-\alpha)(1-\rho)-\Theta \alpha \rho}{2(\Gamma+2)},
$$

there exist constants $\vartheta=\vartheta(\Theta, \rho, \gamma, \alpha)>\alpha(1-\rho), C_{\vartheta}>0$ and $\varepsilon_{0}>0$, which satisfy for all $0<\varepsilon \leqslant \varepsilon_{0}$

$$
\mathbb{P}\left(\exists x \in D^{ \pm}\left(\varepsilon^{\gamma}\right): \sup _{s \in\left[0, T_{1}\right]}\left|Y^{\varepsilon}(s ; x)-u(s ; x)\right|_{\infty} \geqslant(1 / 2) \varepsilon^{2 \gamma}\right) \leqslant C_{\vartheta} \varepsilon^{\vartheta} .
$$

Proof. Let $\Gamma>0$ large enough such that the hypotheses of Lemma 3.2 are satisfied. Then with the given constants there exist constants $C_{\theta}>0$ and $\varepsilon_{0}>0$ such that for $0<\varepsilon \leqslant \varepsilon_{0}$

$$
\begin{aligned}
& \mathbb{P}\left(\exists x \in D^{ \pm}\left(\varepsilon^{\gamma}\right): \sup _{s \in\left[0, T_{1}\right]}\left|Y^{\varepsilon}(s ; x)-u(s ; x)\right|_{\infty} \geqslant(1 / 2) \varepsilon^{2 \gamma}\right) \\
& \begin{aligned}
\leqslant & \mathbb{P}\left(\exists x \in D^{ \pm}\left(\varepsilon^{\gamma}\right): \sup _{s \in[0, t]}\left|Y^{\varepsilon}(s ; x)-u(s ; x)\right|_{\infty} \geqslant(1 / 2) \varepsilon^{2 \gamma}\right) \beta_{\varepsilon} e^{-\beta_{\varepsilon} t} \mathrm{~d} t \\
& \leqslant C_{\theta} \varepsilon^{2-2(\Gamma+2) \gamma-(2-(1-\Theta) \alpha) \rho-\alpha \rho} .
\end{aligned}
\end{aligned}
$$

Fix $\vartheta=2-2(\Gamma+2) \gamma-(2-(1-\Theta) \alpha) \rho-\alpha \rho$. One checks that $\vartheta>\alpha(1-\rho)$.

For $x \in D^{ \pm}\left(\varepsilon^{\gamma}\right)$ define the small perturbation event

$$
E_{x}:=\left\{\sup _{s \in\left[0, T_{1}\right]}\left|Y^{\varepsilon}(s ; x)-u(s ; x)\right|_{\infty} \leqslant(1 / 2) \varepsilon^{2 \gamma}\right\} .
$$

Corollary 3.5. Given the assumptions of Proposition 3.4 there is a constant $\vartheta=\vartheta(\alpha, \Theta, \gamma, \rho)$ with $\vartheta>\alpha(1-\rho), C_{\vartheta}>0$, and $\varepsilon_{0}>0$ such that for all $0<\varepsilon \leqslant \varepsilon_{0}$

$$
\mathbb{E}\left[\sup _{x \in D^{ \pm}\left(\varepsilon^{\gamma}\right)} \mathbf{1}\left(E_{x}^{c}\right)\right] \leqslant C_{\vartheta} \varepsilon^{\vartheta}
$$

Corollary 3.6. Let $C>0$, and let the assumptions of Proposition 3.4 be satisfied. Then there is a constant $\varepsilon_{0}>0$ such that for all $0<\varepsilon \leqslant \varepsilon_{0}, \theta>-1$

$$
\mathbb{E}\left[e^{-\theta \lambda^{ \pm}(\varepsilon) T_{1}} \sup _{x \in D^{ \pm}\left(\varepsilon^{\gamma}\right)} \mathbf{1}\left(E_{x}^{c}\right)\right] \leqslant C\left(\frac{\beta_{\varepsilon}}{\beta_{\varepsilon}+\theta \lambda^{ \pm}(\varepsilon)}\right) \frac{\lambda^{ \pm}(\varepsilon)}{\beta_{\varepsilon}} .
$$




\section{Asymptotic first exit times}

In this section we derive estimates on exit events which then enable us to obtain upper and lower bounds for the Laplace transform of the exit times in the small noise limit.

\subsection{Estimates of Exit Events by Large Jump and Perturbation Events}

To this end, in this subsection we first estimate exit events of $X^{\varepsilon}$ by large jump exits on the one hand, and small deviations on the other hand. Denote the shift by time $t$ on the space of trajectories by $\theta_{t}, t \geqslant 0$. For any $k \in \mathbb{N}, t \in\left[0, t_{k}\right], x \in H$ we have

$$
X^{\varepsilon}\left(t+T_{k-1} ; x\right)=Y^{\varepsilon}\left(t ; X^{\varepsilon}(0 ; x)\right) \circ \theta_{T_{k-1}}+\varepsilon W_{k} \mathbf{1}\left\{t=t_{k}\right\} .
$$

In the following two lemmas we estimate certain events connecting the behavior of $X^{\varepsilon}$ in the domains of the type $D^{ \pm}\left(\varepsilon^{\gamma}\right)$ with the large jumps $\eta^{\varepsilon}$ in the reshifted domains of the type $D_{0}^{ \pm}\left(\varepsilon^{\gamma}\right)$. We introduce for $\varepsilon>0$ and $x \in \tilde{D}^{ \pm}\left(\varepsilon^{\gamma}\right)$ the events

$$
\begin{aligned}
& A_{x}:=\left\{Y^{\varepsilon}(s ; x) \in D^{ \pm}\left(\varepsilon^{\gamma}\right) \text { for } s \in\left[0, T_{1}\right] \text { and } Y^{\varepsilon}\left(T_{1} ; x\right)+\varepsilon W_{1} \in D^{ \pm}\left(\varepsilon^{\gamma}\right)\right\}, \\
& B_{x}:=\left\{Y^{\varepsilon}(s ; x) \in D^{ \pm}\left(\varepsilon^{\gamma}\right) \text { for } s \in\left[0, T_{1}\right] \text { and } Y^{\varepsilon}\left(T_{1} ; x\right)+\varepsilon W_{1} \notin D^{ \pm}\left(\varepsilon^{\gamma}\right)\right\}, \\
& C_{x}:=\left\{Y^{\varepsilon}(s ; x) \in D^{ \pm}\left(\varepsilon^{\gamma}\right) \text { f. } s \in\left[0, T_{1}\right] \text { a. } Y^{\varepsilon}\left(T_{1} ; x\right)+\varepsilon W_{1} \in D^{ \pm}\left(\varepsilon^{\gamma}\right) \backslash \tilde{D}^{ \pm}\left(\varepsilon^{\gamma}\right)\right\}, \\
& A_{x}^{-}:=\left\{Y^{\varepsilon}(s ; x) \in D^{ \pm}\left(\varepsilon^{\gamma}\right) \text { for } s \in\left[0, T_{1}\right] \text { and } Y^{\varepsilon}\left(T_{1} ; x\right)+\varepsilon W_{1} \in \tilde{D}^{ \pm}\left(\varepsilon^{\gamma}\right)\right\}
\end{aligned}
$$

We exploit the definitions of the reduced domains of attraction in order to obtain estimates of solution path events by events only depending on the driving noise.

Lemma 4.1 (Partial estimates of the major events). Let $T_{\text {rec }}, \kappa>0$ be given by Proposition 2.4 and assume that Hypotheses (H.1) and (H.2) are satisfied. For $\rho \in\left(\frac{1}{2}, 1\right), \gamma \in(0,1-\rho)$ there exists $\varepsilon_{0}>0$ so that the following inequalities hold true for all $0<\varepsilon \leqslant \varepsilon_{0}$ and $x \in D^{ \pm}\left(\varepsilon^{\gamma}\right)$

$$
\begin{aligned}
\text { i) } \mathbf{1}\left(A_{x}\right) \mathbf{1}\left(E_{x}\right) \mathbf{1}\left\{T_{1} \geqslant T_{r e c}+\kappa \gamma|\ln \varepsilon|\right\} \leqslant \mathbf{1}\left\{\varepsilon W_{1} \in D_{0}^{ \pm}\right\}, \\
\text {ii) } \mathbf{1}\left(B_{x}\right) \mathbf{1}\left(E_{x}\right) \mathbf{1}\left\{T_{1} \geqslant T_{r e c}+\kappa \gamma|\ln \varepsilon|\right\} \leqslant \mathbf{1}\left\{\varepsilon W_{1} \notin D_{0}^{ \pm}\left(\varepsilon^{\gamma}, \varepsilon^{2 \gamma}\right)\right\}, \\
\text { iii) } \mathbf{1}\left(C_{x}\right) \mathbf{1}\left(E_{x}\right) \mathbf{1}\left\{T_{1} \geqslant T_{r e c}+\kappa \gamma|\ln \varepsilon|\right\} \leqslant \mathbf{1}\left\{\varepsilon W_{1} \in D_{0}^{*}\left(\varepsilon^{\gamma}\right)\right\} .
\end{aligned}
$$

Additionally, for $x \in D^{ \pm}\left(\varepsilon^{\gamma}\right)$ we have

$$
\text { iv) } \begin{aligned}
\mathbf{1}\left(B_{x}\right) \mathbf{1}\left(E_{x}\right) \mathbf{1}\left\{\left\|\varepsilon W_{1}\right\| \leqslant(1 / 2) \varepsilon^{2 \gamma}\right\} \mathbf{1}\left\{T_{1}>T_{\text {rec }}+\kappa \gamma|\ln \varepsilon|\right\}=0, \\
\text { v) } \mathbf{1}\left(C_{x}\right) \mathbf{1}\left(E_{x}\right) \mathbf{1}\left\{\left\|\varepsilon W_{1}\right\| \leqslant(1 / 2) \varepsilon^{2 \gamma}\right\} \mathbf{1}\left\{T_{1} \geqslant T_{r e c}+\kappa \gamma|\ln \varepsilon|\right\}=0 .
\end{aligned}
$$

In the opposite sense for $x \in \tilde{D}^{ \pm}\left(\varepsilon^{\gamma}\right)$

$$
\begin{aligned}
& \text { vi) } \mathbf{1}\left(E_{x}\right) \mathbf{1}\left\{T_{1} \geqslant T_{r e c}+\kappa \gamma|\ln \varepsilon|\right\} \mathbf{1}\left\{\varepsilon W_{1} \notin D_{0}^{ \pm}\right\} \leqslant \mathbf{1}\left(B_{x}\right), \\
& \text { vii) } \mathbf{1}\left(E_{x}\right) \mathbf{1}\left\{T_{1} \geqslant T_{r e c}+\kappa \gamma|\ln \varepsilon|\right\} \mathbf{1}\left\{\varepsilon W_{1} \in D_{0}^{ \pm}\left(\varepsilon^{\gamma}, \varepsilon^{2 \gamma}, \varepsilon^{2 \gamma}\right)\right\} \leqslant \mathbf{1}\left(A_{x}^{-}\right) .
\end{aligned}
$$

With the help of Lemma 4.1 we can show the following crucial estimates.

Lemma 4.2 (Full estimates of the major events). Let $T_{\text {rec }}, \kappa>0$ be given by Proposition 2.4 and Hypotheses (H.1) and (H.2) be satisfied. For $\rho \in\left(\frac{1}{2}, 1\right), \gamma \in(0,1-\rho)$ there exists $\varepsilon_{0}>0$ such that the following inequalities hold true for all $0<\varepsilon \leqslant \varepsilon_{0}, \kappa>0$ and $x \in D^{ \pm}\left(\varepsilon^{\gamma}\right)$

$$
\begin{aligned}
\text { ix }) \mathbf{1}\left(A_{x}\right) & \leqslant \mathbf{1}\left\{\varepsilon W_{1} \in D_{0}^{ \pm}\right\}+\mathbf{1}\left\{\left\|\varepsilon W_{1}\right\|>\frac{1}{2} \varepsilon^{2 \gamma}\right\} \mathbf{1}\left\{T_{1}<T_{r e c}+\kappa \gamma|\ln \varepsilon|\right\}+\mathbf{1}\left(E_{x}^{c}\right), \\
\text { x) } \mathbf{1}\left(B_{x}\right) & \leqslant \mathbf{1}\left\{\varepsilon W_{1} \notin D_{0}^{ \pm}\left(\varepsilon^{\gamma}, \varepsilon^{2 \gamma}\right)\right\}+\mathbf{1}\left\{T_{1}<T_{r e c}+\kappa \gamma|\ln \varepsilon|\right\}+\mathbf{1}\left(E_{x}^{c}\right),
\end{aligned}
$$




$$
\begin{aligned}
\text { xi) } & \sup _{y \in \tilde{D}^{ \pm}\left(\varepsilon^{\gamma}\right)} \mathbf{1}\left\{Y^{\varepsilon}(s ; y) \notin D^{ \pm}\left(\varepsilon^{\gamma}\right) \text { for some } s \in\left(0, T_{1}\right)\right\} \leqslant \sup _{y \in \tilde{D}^{ \pm}\left(\varepsilon^{\gamma}\right)} \mathbf{1}\left(E_{y}^{c}\right), \\
\text { xii) } & \mathbf{1}\left(A_{x}\right) \mathbf{1}\left\{Y^{\varepsilon}\left(s ; X^{\varepsilon}(0, x)\right) \circ \theta_{T_{1}} \notin D^{ \pm}\left(\varepsilon^{\gamma}\right) \text { for some } s \in\left(0, T_{1}\right)\right\} \\
\leqslant & \mathbf{1}\left\{\varepsilon W_{1} \in D_{0}^{*}\left(\varepsilon^{\gamma}\right)\right\}+\mathbf{1}\left\{T_{1}<T_{r e c}+\kappa \gamma|\ln \varepsilon|\right\}+\sup _{y \in \tilde{D}^{ \pm}\left(\varepsilon^{\gamma}\right)} \mathbf{1}\left(E_{y}^{c}\right) \circ \theta_{T_{1}}+\mathbf{1}\left(E_{x}^{c}\right) .
\end{aligned}
$$

In the opposite sense for $x \in \tilde{D}^{ \pm}\left(\varepsilon^{\gamma}\right)$

$$
\begin{aligned}
& \text { xiii) } \mathbf{1}\left(A_{x}^{-}\right) \geqslant \mathbf{1}\left\{\varepsilon W_{1} \in D_{0}^{ \pm}\left(\varepsilon^{\gamma}, \varepsilon^{2 \gamma}, \varepsilon^{2 \gamma}\right)\right\}-\mathbf{1}\left\{T_{1}<T_{r e c}+\kappa \gamma|\ln \varepsilon|\right\}-2 \mathbf{1}\left(E_{x}^{c}\right), \\
& \text { xiv) } \mathbf{1}\left(B_{x}\right) \geqslant \mathbf{1}\left\{\varepsilon W_{1} \notin D_{0}^{ \pm}\right\}\left(1-\mathbf{1}\left\{T_{1}<T_{r e c}+\kappa \gamma|\ln \varepsilon|\right\}\right)-\mathbf{1}\left(E_{x}^{c}\right) .
\end{aligned}
$$

The next lemma ensures that after having relaxed to $B_{\varepsilon^{2 \gamma}}\left(\phi^{ \pm}\right)$the solution $X^{\varepsilon}$ jumps close to the separatrix only with negligible probability for $\varepsilon \rightarrow 0+$.

Lemma 4.3 (Asymptotic behavior of large jump events). Let Hypotheses (H.1) and (H.2) be satisfied and $1 / 2<\rho<1-2 \gamma$. Then for any $C>0$ there is $\varepsilon_{0}=\varepsilon_{0}(C)>0$ such that for all $0<\varepsilon \leqslant \varepsilon_{0}$

$$
\begin{aligned}
& \text { I) }\left(\frac{\mu\left(\left(D_{0}^{ \pm}\right)^{c}\right)}{\mu\left(B_{1}^{c}(0)\right)}-C\right) \varepsilon^{\alpha(1-\rho)} \leqslant \frac{\lambda^{ \pm}(\varepsilon)}{\beta_{\varepsilon}} \leqslant\left(\frac{\mu\left(\left(D_{0}^{ \pm}\right)^{c}\right)}{\mu\left(B_{1}^{c}(0)\right)}+C\right) \varepsilon^{\alpha(1-\rho)}, \\
& \text { II) } \mathbb{P}\left(\left\|\varepsilon W_{1}\right\|>(1 / 2) \varepsilon^{2 \gamma}\right) \leqslant 4 \varepsilon^{\alpha(1-\rho-2 \gamma)}, \\
& \text { III) } \mathbb{P}\left(\varepsilon W_{1} \in\left(\tilde{D}_{0}^{ \pm}\left(\varepsilon^{\gamma}\right)\right)^{c}\right) \leqslant(1+C) \frac{\lambda^{ \pm}(\varepsilon)}{\beta_{\varepsilon}}, \\
& \text { IV) } \mathbb{P}\left(\varepsilon W_{1} \in D_{0}^{*}\left(\varepsilon^{\gamma}\right)\right) \leqslant C \frac{\lambda^{ \pm}(\varepsilon)}{\beta_{\varepsilon}}, \\
& \text { V) } \mathbb{P}\left(\varepsilon W_{1} \in D_{0}^{c}\left(\varepsilon^{\gamma}, \varepsilon^{2 \gamma}, \varepsilon^{2 \gamma}\right)\right) \leqslant(1+C) \frac{\lambda^{ \pm}(\varepsilon)}{\beta_{\varepsilon}} .
\end{aligned}
$$

A detailed proof is given in [4].

\subsection{Asymptotic Exit Times from Reduced Domains of Attraction}

We next exploit the estimates obtained in the previous subsection and combine them with the small deviations result of section 3 , to identify the exit times from the reduced domains of attraction with large jumps from small neighborhoods of the stable equilibria that are large enough to cross the separatrix.

Proposition 4.4 (The upper estimate). Let (H.1) and (H.2) be satisfied. Then for all $\theta>-1$ and $C \in(0,1+\theta)$ there is $\varepsilon_{0}=\varepsilon_{0}(\theta)>0$ such that for $0<\varepsilon \leqslant \varepsilon_{0}$

$$
\mathbb{E}\left[\sup _{x \in \tilde{D}^{ \pm}\left(\varepsilon^{r}\right)} \exp \left(-\theta \lambda^{ \pm}(\varepsilon) \tau_{x}^{ \pm}(\varepsilon)\right)\right] \leqslant \frac{1+C}{1+\theta-C} .
$$

Proof. By (H.2) $\Gamma>0$ can be chosen large enough to fulfill the hypotheses of Proposition 3.4. Let $C>0$ be given. We drop the superscript \pm . Since the jumps of the noise process $L$ exceed any fixed barrier $\mathbb{P}$-a.s., $\tau_{x}(\varepsilon)$ is $\mathbb{P}$-a.s. finite. Therefore we may rewrite the Laplace transform of $\tau_{x}(\varepsilon)$ 
for $\varepsilon>0$, giving

$$
\begin{aligned}
& \mathbb{E}\left[\sup _{x \in \tilde{D}\left(\varepsilon^{\gamma}\right)} e^{\left.-\theta \lambda(\varepsilon) \tau_{x}(\varepsilon)\right]=\sum_{k=1}^{\infty}}\right.\left(\mathbb{E}\left[e^{-\theta \lambda(\varepsilon) T_{k}} \sup _{x \in \tilde{D}\left(\varepsilon^{\gamma}\right)} \mathbf{1}\left\{\tau_{x}(\varepsilon)=T_{k}\right\}\right]\right. \\
&\left.+\mathbb{E}\left[\sup _{x \in \tilde{D}\left(\varepsilon^{\gamma}\right)} e^{-\theta \lambda(\varepsilon) \tau_{x}(\varepsilon)} \mathbf{1}\left\{\tau_{x}(\varepsilon) \in\left(T_{k-1}, T_{k}\right)\right\}\right]\right)=I_{1}+I_{2}
\end{aligned}
$$

Using the strong Markov property, the independence and stationarity of the increments of the large jumps $W_{i}$ we obtain for $k \geqslant 1$

$$
\begin{aligned}
\mathbb{E}\left[e ^ { - \theta \lambda ( \varepsilon ) T _ { k } } \operatorname { s u p } _ { x \in \tilde { D } ( \varepsilon ^ { \gamma } ) } 1 \left\{\tau_{x}(\varepsilon)\right.\right. & \left.\left.=T_{k}\right\}\right] \\
& \leqslant\left(\mathbb{E}\left[e^{-\theta \lambda(\varepsilon) T_{1}} \sup _{y \in D\left(\varepsilon^{\gamma}\right)} 1\left(A_{y}\right)\right]\right)^{k-1} \mathbb{E}\left[e^{-\theta \lambda(\varepsilon) T_{1}} \sup _{y \in D\left(\varepsilon^{r}\right)} 1\left(B_{y}\right)\right] .
\end{aligned}
$$

In the subsequent Claims 1-4 we estimate the preceding factors with the help of Lemma 4.2.

Claim 1: There exists $\varepsilon_{0}>0$ such that for all $0<\varepsilon \leqslant \varepsilon_{0}$

$$
\mathbb{E}_{x}\left[e^{-\theta \lambda(\varepsilon) T_{1}} \sup _{y \in D\left(\varepsilon^{\gamma}\right)} \mathbf{1}\left(A_{y}\right)\right] \leqslant \frac{\beta_{\varepsilon}}{\beta_{\varepsilon}+\theta \lambda(\varepsilon)}\left(1-\frac{\lambda(\varepsilon)}{\beta_{\varepsilon}}(1-C / 5)\right) .
$$

In fact: in the inequality of Lemma $4.2 i x$ ) we can pass to the supremum in $y \in D\left(\varepsilon^{\gamma}\right)$, and integrate to obtain, using the independence of $\left(W_{i}\right)_{i \in \mathbb{N}}$ and $\left(T_{i}\right)_{i \in \mathbb{N}}$

$$
\begin{aligned}
& \mathbb{E}\left[e^{-\theta \lambda(\varepsilon) T_{1}} \sup _{y \in D\left(\varepsilon^{\gamma}\right)} \mathbf{1}\left(A_{y}\right)\right] \\
& \leqslant \mathbb{E}\left[e^{-\theta \lambda(\varepsilon) T_{1}} \mathbf{1}\left\{T_{1}<T_{r e c}+\kappa \gamma|\ln \varepsilon|\right\}\right] \mathbb{P}\left(\varepsilon\left\|W_{1}\right\|>(1 / 2) \varepsilon^{2 \gamma}\right) \\
&+ \mathbb{E}\left[e^{-\theta \lambda(\varepsilon) T_{1}}\right] \mathbb{P}\left(\varepsilon W_{1} \in D_{0}\right)+\mathbb{E}\left[e^{-\theta \lambda(\varepsilon) T_{1}} \sup _{y \in D\left(\varepsilon^{\gamma}\right)} \mathbf{1}\left(E_{y}^{c}\right)\right] \\
&=: K_{1} K_{2}+K_{3} K_{4}+K_{5} .
\end{aligned}
$$

The terms $K_{1}, K_{3}$ and $K_{4}$ can be calculated explicitly, for $K_{2}$ we apply Lemma $4.3 I I$ ). For $K_{5}$ we use Corollary 3.6 and Lemma $4.3 \mathrm{I}$ ) ensuring that there is $\varepsilon_{0}$ so that we have for $0<\varepsilon \leqslant \varepsilon_{0}$

$$
K_{5} \leqslant C / 10 \frac{\beta_{\varepsilon}}{\beta_{\varepsilon}+\theta \lambda(\varepsilon)} \frac{\lambda(\varepsilon)}{\beta_{\varepsilon}} .
$$

Claim 2: There is $\varepsilon_{0}>0$ such that for all $0<\varepsilon \leqslant \varepsilon_{0}$

$$
\mathbb{E}\left[e^{-\theta \lambda(\varepsilon) T_{1}} \sup _{y \in D\left(\varepsilon^{\gamma}\right)} \mathbf{1}(B(y))\right] \leqslant(1+C) \frac{\beta_{\varepsilon}}{\beta_{\varepsilon}+\theta \lambda(\varepsilon)} \frac{\lambda(\varepsilon)}{\beta_{\varepsilon}} .
$$


Indeed, in a similar manner and with the help of Lemma $4.2 x$ ) and Lemma 4.3 III) we obtain that there is $\varepsilon_{0}>0$ such that for all $0<\varepsilon \leqslant \varepsilon_{0}$

$$
\mathbb{E}\left[e^{-\theta \lambda(\varepsilon) T_{1}} \sup _{y \in D\left(\varepsilon^{\gamma}\right)} \mathbf{1}(B(y))\right] \leqslant(1+C) \frac{\beta_{\varepsilon}}{\beta_{\varepsilon}+\theta \lambda(\varepsilon)} \frac{\lambda(\varepsilon)}{\beta_{\varepsilon}} .
$$

In order to treat the summands of the second sum of (4.10) we have to distinguish the cases $\theta \geqslant 0$ and $\theta \in(-1,0)$, as well as $k=1$ and $k \geqslant 2$. Let us first discuss the case $\theta \geqslant 0$.

Claim 3: There is $\varepsilon_{0}>0$ such that for all $0<\varepsilon \leqslant \varepsilon_{0}$

$$
\mathbb{E}\left[\sup _{x \in \tilde{D}\left(\varepsilon^{\gamma}\right)} e^{-\theta \lambda(\varepsilon) \tau_{x}(\varepsilon)} \mathbf{1}\left\{\tau_{x}(\varepsilon) \in\left(0, T_{1}\right)\right\}\right] \leqslant C / 5\left(\frac{\beta_{\varepsilon}}{\beta_{\varepsilon}+\theta \lambda(\varepsilon)}\right) \frac{\lambda(\varepsilon)}{\beta_{\varepsilon}} .
$$

This statement is proved by means of Lemma $4.2 x i$ ) and Corollary 3.5.

Claim 4: There exists $\varepsilon_{0}>0$ such that for any $k \geqslant 2$

$$
\begin{aligned}
\mathbb{E}\left[\operatorname { s u p } _ { x \in \tilde { D } ( \varepsilon ^ { \prime } ) } e ^ { - \theta \lambda ( \varepsilon ) \tau _ { x } ( \varepsilon ) } \mathbf { 1 } \left\{\tau_{x}(\varepsilon)\right.\right. & \left.\left.\in\left(T_{k-1}, T_{k}\right)\right\}\right] \\
\leqslant & \left(\frac{\beta_{\varepsilon}}{\beta_{\varepsilon}+\theta \lambda(\varepsilon)}\left(1-\frac{\lambda(\varepsilon)}{\beta_{\varepsilon}}(1-C / 5)\right)\right)^{k-2} C / 5 \frac{\beta_{\varepsilon}}{\beta_{\varepsilon}+\theta \lambda(\varepsilon)} \frac{\lambda(\varepsilon)}{\beta_{\varepsilon}} .
\end{aligned}
$$

To show this, we use the strong Markov property and Lemma 4.2 xii), as in the estimate for the first summand to get for $k \geqslant 2$ and $\theta \geqslant 0$

$$
\begin{aligned}
\mathbb{E}\left[\operatorname { s u p } _ { x \in \tilde { D } ( \varepsilon ^ { \gamma } ) } e ^ { - \theta \lambda ( \varepsilon ) T _ { k - 1 } } \mathbf { 1 } \left\{\tau_{x}(\varepsilon)\right.\right. & \left.\left.\left(T_{k-1}, T_{k}\right)\right\}\right] \\
& \leqslant\left(\mathbb{E}\left[e^{-\theta \lambda(\varepsilon) T_{1}} \sup _{y \in D\left(\varepsilon^{\gamma}\right)} \mathbf{1}\left(A_{y}\right)\right]\right)^{k-2}\left(K_{3} K_{9}+K_{1}+2 K_{5}\right) .
\end{aligned}
$$

Lemma $4.2 \mathrm{xii}$ ) and Lemma $4.3 \mathrm{IV}$ ) provide the existence of $\varepsilon_{0}>0$ such that for $0<\varepsilon \leqslant \varepsilon_{0}$

$$
K_{9}=\mathbb{P}\left(\varepsilon W_{1} \in D_{0}^{*}\left(\varepsilon^{\gamma}\right)\right) \leqslant C / 20 \frac{\lambda(\varepsilon)}{\beta_{\varepsilon}} .
$$

It remains to discuss the case $\theta \in(-1,0)$ in a similar way. This is detailed in [4].

Combining Claims 1-4 we finally find an $\varepsilon_{0}>0$ such that for (4.10) and all $0<\varepsilon \leqslant \varepsilon_{0}$

$$
\begin{aligned}
\mathbb{E}\left[\sup _{x \in \tilde{D}\left(\varepsilon^{\prime}\right)} e^{-\theta \lambda(\varepsilon) \tau_{x}(\varepsilon)}\right] & \\
\leqslant(1+(2 / 5) C) \frac{\lambda(\varepsilon)}{\beta_{\varepsilon}} \frac{\beta_{\varepsilon}}{\beta_{\varepsilon}+\theta \lambda(\varepsilon)} \sum_{k=0}^{\infty}\left(\frac{\beta_{\varepsilon}}{\beta_{\varepsilon}+\theta \lambda(\varepsilon)}\left(1-\frac{\lambda(\varepsilon)}{\beta_{\varepsilon}}(1-C / 5)\right)\right)^{k} & \leqslant \frac{1+C}{\theta+(1-C)} .
\end{aligned}
$$

The series converges if and only if $C<\theta+1$. 
Proposition 4.5 (The lower estimate). Assume that Hypotheses (H.1) and (H.2) are satisfied. Then for all $\theta>-1$ and $C \in(0,1+\theta)$ there is $\varepsilon_{0}=\varepsilon_{0}(\theta)>0$ such that for all $0<\varepsilon \leqslant \varepsilon_{0}$

$$
\mathbb{E}\left[\inf _{x \in \tilde{D}^{ \pm}\left(\varepsilon^{r}\right)} \exp \left(-\theta \lambda^{ \pm}(\varepsilon) \tau_{x}^{ \pm}(\varepsilon)\right)\right] \geqslant \frac{1+C}{1+\theta-C} .
$$

Proof. Again we omit the superscript \pm and fix $\Gamma>0$ large enough due to (H.2). Omitting the term $I_{2}$ in equation (4.10), we obtain the estimate

$$
\begin{aligned}
\mathbb{E}\left[\inf _{x \in \tilde{D}\left(\varepsilon^{\gamma}\right)} e^{-\theta \lambda(\varepsilon) \tau_{x}(\varepsilon)}\right] & \\
& \geqslant \sum_{k=1}^{\infty}\left(\mathbb{E}\left[e^{-\theta \lambda(\varepsilon) T_{1}} \inf _{y \in \tilde{D}\left(\varepsilon^{\gamma}\right)} \mathbf{1}\left(A_{y}^{-}\right)\right]\right)^{k-1} \mathbb{E}\left[e^{-\theta \lambda(\varepsilon) T_{1}} \inf _{y \in \tilde{D}\left(\varepsilon^{\gamma}\right)} \mathbf{1}\left(B_{y}\right)\right] .
\end{aligned}
$$

We treat the terms appearing in (4.13) in a similar way as for the upper estimate.

Claim 1: There is $\varepsilon_{0}>0$ such that for all $0<\varepsilon \leqslant \varepsilon_{0}$

$$
\mathbb{E}\left[e^{-\theta \lambda(\varepsilon) T_{1}} \inf _{x \in \tilde{D}\left(\varepsilon^{\gamma}\right)} \mathbf{1}\left(A_{x}^{-}\right)\right] \geqslant \frac{\beta_{\varepsilon}}{\beta_{\varepsilon}+\theta \lambda(\varepsilon)}\left(1-(1+C) \frac{\lambda(\varepsilon)}{\beta_{\varepsilon}}\right) .
$$

To prove this, we apply Lemma $4.1 x i i i)$, take the infimum over $y \in \tilde{D}\left(\varepsilon^{\gamma}\right)$ and integrate to get

$$
\begin{aligned}
\mathbb{E}\left[e^{-\theta \lambda(\varepsilon) T_{1}} \inf _{y \in \tilde{D}\left(\varepsilon^{\gamma}\right)}\right. & \left.1\left(A_{y}^{-}\right)\right] \\
& =K_{3}\left(1-\mathbb{P}\left(W_{1} \in(1 / \varepsilon) D_{0}^{c}\left(\varepsilon^{\gamma}, \varepsilon^{2 \gamma}, \varepsilon^{2 \gamma}\right)\right)\right)-K_{1}-2 K_{5},
\end{aligned}
$$

where $K_{1}, K_{3}, K_{5}$ have the same meaning as in the proof of Proposition 4.4 and are treated identically.

By Lemma $4.3 \mathrm{~V}$ ) there exists $\varepsilon_{0}>0$ such that for $0<\varepsilon \leqslant \varepsilon_{0}$

$$
\mathbb{P}\left(\varepsilon W_{1} \in D_{0}^{c}\left(\varepsilon^{\gamma}, \varepsilon^{2 \gamma}, \varepsilon^{2 \gamma}\right)\right) \leqslant(1+C / 5) \frac{\lambda(\varepsilon)}{\beta_{\varepsilon}} .
$$

Claim 2: There is $\varepsilon_{0}>0$ such that for $0<\varepsilon \leqslant \varepsilon_{0}$

$$
\mathbb{E}\left[e^{-\theta \lambda(\varepsilon) T_{1}} \inf _{y \in \tilde{D}_{0}\left(\varepsilon^{\gamma}\right)} \mathbf{1}\left(B_{y}\right)\right] \geqslant \frac{\beta_{\varepsilon}}{\theta \lambda(\varepsilon)+\beta_{\varepsilon}}\left((1-C) \frac{\lambda(\varepsilon)}{\beta_{\varepsilon}}\right) .
$$

Here we exploit Lemma $4.2 x i v$ ). Finally combining Claim 1 and Claim 2 we obtain

$$
\begin{aligned}
\mathbb{E}\left[\inf _{x \in \tilde{D}\left(\varepsilon^{r}\right)} e^{-\theta \lambda(\varepsilon) \tau_{x}(\varepsilon)}\right] \geq & \\
\geqslant \sum_{k=1}^{\infty}\left(\frac{\beta_{\varepsilon}}{\beta_{\varepsilon}+\theta \lambda(\varepsilon)}\left(1-(1+C) \frac{\lambda(\varepsilon)}{\beta_{\varepsilon}}\right)\right)^{k-1} & \frac{\beta_{\varepsilon}}{\theta \lambda(\varepsilon)+\beta_{\varepsilon}}\left((1-C) \frac{\lambda(\varepsilon)}{\beta_{\varepsilon}}\right) \\
& =\frac{\lambda(\varepsilon)(1-C)}{\theta \lambda(\varepsilon)-(1+C) \lambda(\varepsilon)}=\frac{1-C}{\theta+1+C}
\end{aligned}
$$


The series converges if and only if $-(1+C)<\theta$.

\section{References}

[1] L. Arnold. Hasselmann's Program revisited: The analysis of stochasticity in deterministic climate models, in: P. Imkeller and J.-S. von Storch (eds): Stochastic Climate Models. Progress in Probability Birkhauser, Basel, 2001. MR1948294

[2] N. H. Bingham, C. M. Goldie, J. L. Teugels. Regular variation, Cambridge University Press, Cambridge, 1987. MR0898871

[3] M. Claussen, L. A. Mysak, A. J. Weaver, M. Crucix, T. Fichefet, M.-F. Loutre, S. L. Weber, J. Alcamo, V. A. Alexeev, A. Berger, R. Calov, A. Ganopolski, H. Goosse, G. Lohmann, F. Lunkeit, I. I. Mokhov, V. Petoukhov, P. Stone, Z. Wang, Earth System Models of Iintermediate Complexity: Closing the gap in the spectrum of climate system models, Climate Dynamics, 18, (2002), 579-586, DOI 10.1007/s00382-001-0200-1.

[4] A. Debussche, M. Högele, P. Imkeller. Metastability for the Chafee-Infante equation with small heavy-tailed Lévy noise, (to appear), 2011.

[5] P. D. Ditlevsen, Observation of a stable noise induced millennial climate changes from an icecore record, Geophysical Research Letters, 26 (10) (1999), 1441-1444.

[6] P. D. Ditlevsen, Anomalous jumping in a double-well potential, Physical Review E, 60(1) (1999), 172-179, 1999.

[7] A. Eden, C. Foias, B. Nicolaenko, R. Temam. Exponential attractors for dissipative evolution equations, Masson, Paris; John Wiley and Sons, Ldt., Chichester, 1994. MR1335230

[8] G. W. Faris, G. Jona-Lasinio. Large fluctuations for a nonlinear heat equation with noise, J. Phys. A 15 (1982), 3025-3055 . MR0684578

[9] V. V. Godovanchuk. Asymptotic probabilities of large deviations due to large jumps of a Markov process (English translation), Theory of Probab Appl. 26 (2) (1981-1982), 314-327. MR0616623

[10] D. Henry. Some infinite-dimensional Morse-Smale systems defined by parabolic partial differential equations, J. Differential Equations, 59 (1985), no 2, 165-205. MR0804887

[11] D. Henry. Geometric theory of semilinear parabolic equations, Lecture notes in Mathematics, 840. Springer-Verlag, Berlin-New York, 1981. MR0610244

[12] H. Hulk and F. Lindskog. Regular variation for measures on metric spaces, Publ. Inst. Math. (Beograd) (N.S.) 80 (2006), no 94, 121-140. MR2281910

[13] P. Imkeller, Energy balance models: viewed from stochastic dynamics, Progr. Probab., 49, $213-$ 240, Basel, 2001. MR1948298

[14] P. Imkeller and I. Pavlyukevich, Metastable behaviour of small noise Lévy-driven diffusions, ESAIM: Prob. Stat., 12 (2008), 412-437. MR2437717 
[15] P. Imkeller and I. Pavlyukevich, First exit times of SDEs driven by stable Lévy processes, Stoch. Proc. Appl., 116 (2006), no 4, 611-642. MR2205118

[16] S. Peszat and J. Zabczyk, Stochastic partial differential equations with Lévy noise (an evolution equation approach), Cambridge University Press, Cambridge, 2007. MR2356959

[17] G. Raugel, Global attractors in partial differential equations, Fiedler, Bernold (ed.), Handbook of dynamical systems. Vol 2, 885-982, North-Holland, Amsterdam, 2002. MR1901068 